\title{
Applicability of Scaling Approach for Analysis of Pyrolysis and Gasification of Porous Structures Composed of Solid Fuel Particles
}

\author{
Aliaksandr Alevanau, Pawel Donaj, Weihong Yang, and Wlodzimierz Blasiak \\ Division of Energy and Furnace Technology, ITM/MSE, KTH, Brinellvägen 23, 10044 Stockholm, Sweden \\ Correspondence should be addressed to Pawel Donaj, pawel@mse.kth.se
}

Received 29 December 2011; Accepted 7 February 2012

Academic Editors: A. Postelnicu and A. Z. Sahin

Copyright (C) 2012 Aliaksandr Alevanau et al. This is an open access article distributed under the Creative Commons Attribution License, which permits unrestricted use, distribution, and reproduction in any medium, provided the original work is properly cited.

Experimental research on the pyrolysis and gasification of randomly packed straw pellets was conducted with an emphasis on the reactive properties of the shrinking porous structure of the pellets. The apparent kinetics of such pyrolysis was approximated by the random pore, grain, and volumetric models. The best approximation results were obtained with the grain and random pore models. The self-organized oscillations of the pellet conversion rate during pyrolysis were observed. Two complementary explanations of the phenomenon are proposed.

\section{Introduction}

Old and modern gasification reactors are fed by various types of fuel, including raw biomass and biomass in pelletized form. Wood chips and chunks of different forms and sizes are also applied, along with a pulverized form of pellets. As the size of a biomass particle decreases, its pyrolysis and gasification occur more quickly. Thus, it may be beneficial for the conditions of industrial scale gasification to feed reactors with a pulverized fuel. However, if one considers a fixed-bed type reactor, the small particles of pulverized fuel may be partially sintered during pyrolysis. The resulting char will have a completely different permeability for gasification agents than that of the pulverized particles in their initially loose state. The release of tars and oils during pyrolysis may also substantially decrease the permeability of the main biomass stock for gasification agents. The bubbling of the particles in fluidized bed-type reactors is among the alternatives that avoid these drawbacks of fixed-bed type reactors. The bubbling, however, has its own disadvantages; for example, it is necessary to prepare particles with a narrow size distribution, and a relatively high flow of a gasification agent is required to maintain bubbling of the particles.
Considering these issues and the additional energy and processing costs required for fuel pulverization, exploiters and developers of industrial scale gasifiers and gasifiers of the types described in [1] may prefer the application of raw biomass, wood pellets, chunks, and chips instead of their pulverized form.

Despite this choice, the developers and exploiters of reactors should consider the dependence of gasification regimes on the evolution of porous structures that are created in the bed. To achieve the optimal regimes, it should be possible to predict the regimes of both pyrolysis and char gasification in a packed bed as they depend on the temperature, gasification agent, and fuel feeding rates. The evolution of porous structures, from the delivery of fresh fuel to the bed to the disappearance of completely gasified char, must be simulated, along with the dynamics of ash-porous structures.

1.1. Literature Review. The best-known analytical results applicable for the approximation of the gasification process in porous structures belong to the random pore (RPM) $[2,3]$ and grain models $(\mathrm{GM})[4,5]$. These models analyze changes in the dynamics of chemical processes as they depend on 
changes in porous structures. A chemical process changes a porous structure, and the dynamics of the process are changed in turn because of the change of the structure. In the particular case of the metallurgical gasification of pellets with metal oxides and the case of the gasification of biomass, the process of gasification agent diffusion is accompanied by various chemical reactions that occur with the components constituting the porous structures. The components change during these reactions, and the possibilities for gasification agents to come into physical contact with these components change accordingly. Because different materials have different internal porosity properties, the models may be differently applicable to different materials. Fermoso et al. [6] compared the applicability of the random pore, grain, and volumetric models for the prediction of the extent of conversion in five different chars. As expected, the random pore model demonstrated the best applicability for such a task. Its advantage over the grain and volumetric models is based on its consideration of the overlapping of pores when their growth competes with the decrease of their number because of coalescence. The grain model considers the porous structure of a solid to consist of nonporous reacting grains with unreacted cores that shrink during gasification. The solid skeletons of the cores may remain, or the entire substance of the cores may undergo phase transition to a gaseous state and diffuse out of the shrinking porous structure. These differences in the chemical and physical mechanisms involved in the gasification process lead to many particular modifications and adaptations of the grain and shrinking core models for various particular cases described in the literature.

Both models have also been unified. Raghunathan and Yang [7] presented a unified approach to predict the conversion curve to $70 \%$ of conversion as a function of the $50 \%$ conversion time for chars with average values for the main parameters of both models (the form factor of a grain model and a structural parameter of a random pore model).

These models were developed and applied mainly for kinetic cases, in which the kinetics of the transfer processes in the porous structure is considered to be significantly faster than the kinetics of the chemical processes in the structure. This condition assumes a relatively small size of the porous structure, which does not create substantial resistance to the transfer processes. These assumptions are applied to the basic studies $[2,4]$ of both models (RPM and GM), respectively. The size of the particles considered in [6] as an appropriate test of the models is the typical size of the particles applied in the majority of devices for thermogravimetric analysis (TGA) (p. size $<150 \mu \mathrm{m}$ ). Such sizes allow the application of a volumetric model, along with the grain and random pore models. The basic assumption of a volumetric model is that the gasification agent has free access to the small particles of the gasified substance.

Fermoso et al. [6] showed that all three models have good applicability to the prediction of the conversion of small particles from four different chars of the five chosen for the experiments. The models were not applicable to the case with the essential presence of catalytic ash particles in the char.

A particle-based model of the char gasification process that accounts for the presence of catalytic ash particles, which may agglomerate during the progress of char gasification, was developed by Yamashita et al. [8]. The experimental works conducted by $\mathrm{Wu}$ et al. [9] and by Song and Kim [10] prove the main conclusions of the simulations in [8] regarding the necessity of considering dynamic effects, which change the char reactivity by the competing effects of deactivation and increasing catalytic activity of the particles present in different types of char. To model these effects better, one may apply the tool and method developed by Wang et al. [11] for fixed-bed reactor simulations, which considers the existence of porous structures in the bed that contain interacting particles of different types and sizes. One such particle type may be the particle of catalytic metals forming the ash particles, whose catalytic action may exhibit nonlinear dynamics during gasification because of their agglomeration and the overall increase of their concentration in the disappearing char.

The case of intentionally added catalysts is considered by Gutfraind and Sheintuch [12]. In this case, the catalyst may form a fractal interface (e.g., on the fractal porous structure of the char). This fractal interface adds additional peculiarities to the consideration of the dynamics of the catalytic effects and their variance with the time of gasification.

The gradual change of the fractal dimension of the porous structures of the fuel particles along the length of a fixed bed should also be considered. This change may be caused by the gradual temperature decrease of gasification agents such as steam if the heat for pyrolysis and char gasification is delivered by the steam itself. The dynamics of the fuel particles delivered into the bed for pyrolysis and subsequent gasification of char may cause the part of the bed filled by char to be substantially longer than the part of the bed filled by the particles undergoing pyrolysis. The parameters of porosity, ash agglomeration, and char defragmentation in the beginning and the end of gasification are not uniform along the whole length of the char-filled part of the bed. The fractal dimension of the internal porous structures in the char may also be nonuniform and may gradually change along the length of this part of the bed (in both updraft and downdraft beds).

Due to latter objectivity, one may consider certain side effects of char gasification on pyrolysis in the bed. These side effects may appear if there is a coincidence of the values and rates of change of the fractal dimensions of the char porous structures in the bed and the changing fractal dimensions of the geodesics, as introduced by Nottale in his theory [13]. For these effects to be experimentally detected, the spatial fractal lines of the geodesics must be spanned along the porous structures made by a fuel in the bed. Another logically deduced requirement is the placement of the origin of the geodesics at the scale of the intermolecular interactions in the hydrocarbons undergoing pyrolysis. If the next scale level to pass by the geodesics is the scale level of the porous structures in the char with their fractal dimensions changing along the bed, there is a hypothetical possibility for the interactions with the geodesics to cause their disturbance along this passage. This disturbance may be transmitted via intermediate scale levels to the scale of the intermolecular interactions in the hydrocarbons undergoing pyrolysis. Such 
a disturbance may destabilize the intermolecular interactions in the hydrocarbons and change their normal reforming process during pyrolysis.

Another type of hypothetical effects of processes like diffusion, heat, and mass transfer was described by Nottale in [14]. These effects are caused by self-organization, which may arise from the action of introduced-by-Nottale generalized quantum potentials that oppose the potentials driving the transfer processes. However, the scale level of porous structures in reactors at which the self-organization may appear is unclear.

We have mentioned the fractal theory [13] developed by Nottale because of the wide presence of fractal porous structures created by fuel and catalysts in gasification reactors. The heat and mass transfer processes are active on these structures. Consequently, it may be reasonable to consider the effects caused by fractality in such reactors. However, these effects may require special conditions for their detection and observation. Their magnitude appears to be essential for the processing of fuels with substantial amounts of catalysts, which is not the case for the majority of biomass types [9].

From a general perspective, the GM and RPM models, which do not consider fractality-related effects, may be fully applicable to grains and pores of completely different scales because the basic processes of the growth and overlapping of pores and the shrinking of unreacted cores are qualitatively the same at different scales. The differences that arise with changes of scale are mainly associated with the resistances to mass and heat transfer in and out of the porous structures. If the kinetics of the chemical processes is much slower than the kinetics of the transfer processes in a particular porous structure, the models are applied in their kinetic form. Otherwise such additions to the models as those published in $[3,5]$ are applied.

For microporous systems, the tradeoff between the diffusion and kinetic control becomes important at elevated temperatures. As the temperature increases, the diffusionrelated control may become substantially weaker than the reaction kinetics control. In such a case, one may apply the models in their kinetic form to relatively large porous structures.

We conducted a test of this assumption [15] during experiments with the gasification of chars that were intentionally obtained from pulverized particles of wood pellets and from whole wood pellets. Different pyrolysis conditions were applied to investigate the dependence of the kinetic parameters on both the scale of the gasified porous structures and the differences of these structures caused by differences in the pyrolysis conditions. It appeared to be possible to obtain relatively stable values of the apparent activation energies in all of the experimental cases. The values were estimated by the application of the volumetric, grain, and random pore models in their kinetic forms. Less accurate fit parameters using the three models mentioned above were obtained with the volumetric model and with all of the models at the lowest experimental temperature $\left(800^{\circ} \mathrm{C}\right)$. The scale levels of the gasified porous structures were the level of particles of pulverized pellets $(0.2-1.0 \mathrm{~mm}$ diameter $)$ and the level of individual wood pellets ( $8 \mathrm{~mm}$ diameter).
With the transition to larger scales, diffusion (which is the main mechanism of reactant transfer in and out of the microporous structures) must be replaced by pressure-driven mass transfer. The reaction kinetics observed at such scales is considered to be the apparent reaction kinetics, which is true if the porous structure is composed of particles with sizes much greater than those commonly accepted for kinetic measurements in TGA devices.

In the case of the random packing scale level in a fixed bed, the porous structure to be changed during the process consists of pellets as building blocks. This structure dynamically changes during pyrolysis. The changes include initial swelling and subsequent shrinkage, which occurs with the release of volatiles. Such shrinkage may be partially analogous to the shrinkage of the microporous structure of the char during its gasification by steam. The latter is successfully modeled by GM and RPM (see [6]). By considering the joint transport of mass and heat by steam or argon moving through the structure of randomly packed pellets to be analogous to the diffusion of a gasification agent into the microporous structure of the char, we may consider both the random pore and grain models to be applicable to the simulation of the process, which dynamically changes the para-meters of the random pellet packing. This consideration assumes the application of the so-called scaling approach. Several examples on the application of this approach to the analysis of self-similar functions and dynamical processes of different spatial and time scales are described in the book by Feder [16] (e.g., the Weierstrass function).

The approach is based on the assumption that similarities exist between the totally different processes if they have some common characteristics in their backgrounds. For example, the ability for self-organization [17] (which creates qualitatively the same phenomena on completely different scales) is one such common characteristic. In the particular case of this work, the common background between the gasification process in porous structures on the microscale and the pyrolysis of randomly packed pellets comprising porous structures on the macro-scale is the dynamical change of the porous structures during the process. The change of the porous structure changes the dynamics of the chemical process that occurs in the structure. The exploration of this change on the macro-scale of an experimental basket with the pellets undergoing pyrolysis is one of the objective tasks of this paper.

1.2. Objective. Because of the described basics for the application of the scaling approach to pyrolysis experiments, the objective of this paper is to test the approach using the random pore, grain, and volumetric models to predict the conversion of randomly packed pellets by pyrolysis in atmosphere of high-temperature argon and steam.

The experimental setup for this task is described in Section 2. The results and discussion for the experiments and simulations are presented in Section 3. 
TABLE 1: The proximate and ultimate analysis of the straw pellets.

\begin{tabular}{|c|c|c|c|c|c|c|}
\hline \multirow{2}{*}{$\begin{array}{l}\text { Analysis } \\
\text { Proximate analysis }\end{array}$} & \multirow[b]{2}{*}{ Symbol } & \multirow[b]{2}{*}{ Unit } & \multicolumn{4}{|c|}{ Conditions } \\
\hline & & & $\begin{array}{c}\text { As received } \\
r\end{array}$ & $\begin{array}{c}\text { Analytical } \\
a\end{array}$ & $\begin{array}{c}\text { Dry } \\
d\end{array}$ & $\begin{array}{c}\text { Dry ash-free } \\
\text { daf }\end{array}$ \\
\hline Analytical moisture (dry air) & $\mathrm{W}$ & $\%$ & 5.6 & 5.8 & - & - \\
\hline Total moisture & $\mathrm{W}_{\mathrm{t}}$ & $\%$ & 10.1 & 5.8 & - & - \\
\hline Ash & A & $\%$ & 3.4 & 3.6 & 3.8 & - \\
\hline Combustible matter & & $\%$ & 86.5 & 90.6 & 96.2 & - \\
\hline Volatiles & V & $\%$ & 69.9 & 73.2 & 77.7 & 80.8 \\
\hline HHV (higher heating value) & $\mathrm{Q}_{\mathrm{s}}$ & $\mathrm{kcal} / \mathrm{kg}$ & 4034 & 4224 & 4486 & 4662 \\
\hline $\mathrm{HHV}$ & $\mathrm{Q}_{\mathrm{s}}$ & $\mathrm{kJ} / \mathrm{kg}$ & 16889 & 17685 & 18780 & 19518 \\
\hline LHV (lower heating value) & $\mathrm{Q}_{\mathrm{i}}$ & $\mathrm{kcal} / \mathrm{kg}$ & 3703 & 3905 & 4183 & 4347 \\
\hline LHV & $\mathrm{Q}_{\mathrm{i}}$ & $\mathrm{kJ} / \mathrm{kg}$ & 15503 & 16348 & 17512 & 18200 \\
\hline \multicolumn{7}{|l|}{ Ultimate analysis } \\
\hline Carbon & $\mathrm{C}_{\mathrm{t}}$ & $\%$ & 41.82 & 43.79 & 46.5 & 48.33 \\
\hline Hydrogen & $\mathrm{H}_{\mathrm{t}}$ & $\%$ & 5.22 & 5.47 & 5.81 & 6.04 \\
\hline Oxygen & $\mathrm{O}$ & $\%$ & 39.02 & 40.86 & 43.39 & 45.9 \\
\hline Nitrogen & $\mathrm{N}$ & $\%$ & 0.43 & 0.45 & 0.48 & 0.5 \\
\hline Sulfur & $S_{t}$ & $\%$ & 0.04 & 0.4 & 0.04 & 0.04 \\
\hline Chlorine & $\mathrm{Cl}$ & $\%$ & 0.075 & 0.079 & 0.084 & \\
\hline Fluor & $\mathrm{F}$ & $\%$ & & & & \\
\hline
\end{tabular}

\section{Experimental Materials and Methods}

Industrially produced straw pellets were chosen for the experiments. Their characterization is presented in Table 1.

Experiments on the pyrolysis of the pellets were conducted in the experimental apparatus depicted in Figure 1.

The apparatus applies the initial heating with ceramic honeycombs (3) by the combustion of methane. Then, the combs are switched to heating the gaseous mixtures passing through their hot ceramics. The initial mixing of gases such as nitrogen and oxygen takes place inside the burner (20). This mixing is controlled by electronic gas flow regulators. Steam to one of the mixing inputs is delivered by a separate steam generator. A basket (18) with dimensions of $3 \times 5 \times 8 \mathrm{~cm}$ is produced from $1 \mathrm{~mm}$ cell wire mesh. It is suspended by a tiny wire (6) from the digital balance (7). The construction of the basket includes a thermocouple that provides a Keithley voltmeter with readings of the temperature inside the basket. The thermocouple (18) is heated by convection of the gas passing through the honey combs, by radiation from the combs and by the thermal insulation of a reactor chamber. The thermocouple shows equilibrium temperature that is approximately 120 degrees higher than the temperature of the gases penetrating the basket. The temperature of the gases flowing through the reactor chamber gradually decreases in the direction of the exhaust (9). The glass window (18) allows manual positioning of the basket in the flow. Its insertion into the reaction chamber occurs after the cessation of combustion, followed by placement of the basket under the hatch (8) and the blowing of nitrogen delivered by the cooling input (5). The remaining flue gases are purged from the combs and the reaction chamber by nitrogen from another regulated input. Then, the gaseous mixture from the inputs begins to flow around the immediately inserted basket. Because the basket is suspended from the digital balances with the attached thermocouple, the readings of the mass loss taken during the reactions inside the basket have some noise. The convection of hot air around the wires from the thermocouple and the variation of the gas pressure in the flow produce instabilities in the weight readings. These instabilities are accounted for by an optimization procedure developed in the Scilab language to estimate the apparent kinetic parameters of pyrolysis with the three kinetic models mentioned above. The algorithm of this estimation is based on a multivariable optimization performed on data from several mass loss curves measured at different temperatures. In the case of the assumed compliance of the pyrolysis kinetics to the kinetics of the first order chemical reaction [18], the algorithm should give approximately the same parameters of the activation energy and frequency factor as a volumetric model at every temperature used for the measurements. If the actual process and parameters from the best approximation by the models do not comply with the Arrhenius law, the algorithm shows this directly and gives the optimal fitting parameters of every model for every measured curve. The short description of the models starts from the basic expressions of the Arrhenius kinetic constant and the differential equations that describe the volumetric model.

The Arrhenius kinetic constant of the reaction (which is calculated by (1)) is applied to differential equation (2) to 


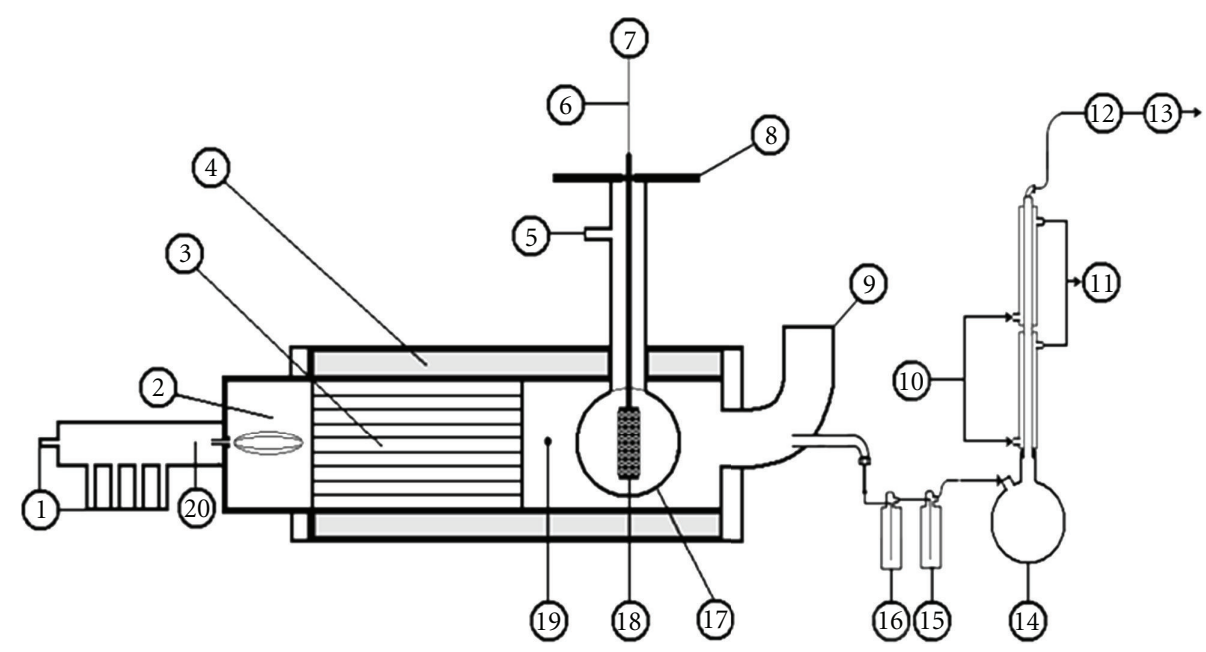

FIGURE 1: Small scale batch-type gasifier: 1: air, methane, oxygen, nitrogen, and steam inputs; 2: combustion chamber; 3: honeycomb; 4: heat insulation; 5: cooling chamber with nitrogen input; 6: tiny wire; 7: digital balance; 8: hatch; 9: exhaust; 10: cold water supply; 11: water sink; 12: flue gas analyzer; 13: GC; 14: water coil cooler with isopropanol trap; 15: first isopropanol trap; 16: water trap; 17: glass window; 18: basket with samples and thermocouple; 19: thermocouple; 20: burner with mixing chamber.

describe the volumetric conversion of a gasified substance with time:

$$
\begin{gathered}
k_{\mathrm{VM}}=k_{0} \mathrm{e}^{-E_{a} / R T}, \\
\frac{\mathrm{d} X}{\mathrm{~d} t}=k_{\mathrm{VM}}(1-X) .
\end{gathered}
$$

The linearized solution of this equation reads

$$
-\ln (1-X)=k_{\mathrm{VM}} t
$$

The existence of a porous structure that undergoes nonlinear evolution is considered in RPM by the application of the structural parameter $\psi$ :

$$
\psi=\frac{4 \pi L_{0}\left(1-\varepsilon_{0}\right)}{S_{0}^{2}} .
$$

Here, $S_{0}$ is the surface of the pores, $L_{0}$ is their length, and $\varepsilon_{0}$ is the solid porosity of the unreacted substance. For a kinetic case, the model reads

$$
\begin{gathered}
\frac{\mathrm{d} X}{\mathrm{~d} t}=k_{\mathrm{RPM}}(1-X) \sqrt{[1-\psi \ln (1-X)]}, \\
\left(\frac{2}{\psi}\right)[\sqrt{[1-\psi \ln (1-X)]}]-1=k_{\mathrm{RPM}} t,
\end{gathered}
$$

$k_{\mathrm{RPM}}$ and $k_{\mathrm{GM}}$ are the Arrhenius kinetic constants calculated in the same way as $k_{\mathrm{VM}}$ for a volumetric model by (1).

The grain model for a particle composed of spherical grains gives the following differential equation for the conversion rate:

$$
\frac{\mathrm{d} X}{\mathrm{~d} t}=k_{\mathrm{GM}}(1-X)^{2 / 3}
$$

This equation has the following linearized solution:

$$
3\left[1-(1-X)^{1 / 3}\right]=k_{\mathrm{GM}} t
$$

The variations of the kinetic parameters obtained for the models at different temperatures show the extent of the deviations of the simulated apparent kinetics from the Arrhenius law. The quality of the fits obtained with the models is estimated using the coefficient of determination calculated by the commonly accepted formula:

$$
R^{2}=\frac{\sum_{i}\left(X_{m i}-\overline{X_{m i}}\right)^{2}-\sum_{i}\left(X_{p i}-X_{m i}\right)^{2}}{\sum_{i}\left(X_{m i}-\overline{X_{m i}}\right)^{2}} .
$$

Here, $X_{m i}$ is calculated from the measurements of mass loss in the basket, and $X_{p i}$ is extent of conversion projected by a model. Because of the low ash content in the pellets, the mass of the ash is neglected in the calculation of $X_{m i}$, which equals the following:

$$
X_{m i}=\frac{m_{0}-m_{i}}{m_{0}}
$$

Here, $m_{0}$ is the initial mass in the basket and $m_{i}$ is its value at the $i$ th measurement, which is taken every second in the applied experimental setup.

The special GM and RPM parameters, which are related to the parameters of the porous structure, are also varied for every temperature because of the expectable initial differences in the parameters of the porous structures made by the pellets in the experimental basket at different temperatures.

The part of the experimental setup that is designed for chemical measurements has been applied for measurements of the compositions of the pyrogases as they depend on the gas/fuel ratios using steam and argon gas. It was assumed that the steam reacts with the tars produced during pyrolysis 
in the random packing of pellets, thus changing the ratios of hydrocarbons measured on output. We reported the results of the gas composition measurements that prove this assumption in [19]. In the current work, we concentrate on approximations of the thermogravimetric results of the same measurements. In such a case, the most important factor, which changes with the change of the steam/fuel ratio and with the switch to argon, is the efficiency of the heat transfer to the randomly packed pellets in the basket. The chemical interaction (or its absence) of the gas with the products of pyrolysis is not considered to be essential for the conversion rate.

The conversion rate prediction by the GM and RPM is expected to follow the changes of the pyrolysis kinetics, which are caused by changes of the porous structure of the randomly packed pellets in the basket. Because of the nature of this random packing, the optimization procedure to obtain the kinetic parameters of the first order pyrolysis reaction and the specific parameters of the models should not be applied to the entire measured mass loss curves. The extent of the approximated conversions should be analyzed up to 50-60\% conversion of the entire mass in the basket, which is less than the extent of conversion achieved by the pellets during their fast pyrolysis (up to $80-90 \%$ of mass, according to the measurements at the highest temperature). This limitation is introduced by the fact that the porous structure does not entirely disappear with the complete conversion of mass, which is expected by the models in the case of, for example, char gasification. The observable change of the porous structure formed by the pellets stops before the final stages of pyrolysis.

The hydrodynamic and temperature conditions in the reaction chamber remained approximately the same with the change of the steam/fuel ratio. The ratio was changed by decreasing the number of pellets in the basket. The pyrolysis in an argon atmosphere with a decreased number of pellets was studied with approximately the same flow parameters ( $1 \mathrm{Nm}^{3} /$ hour $\sim 1.783 \mathrm{~kg} /$ hour, Reynolds number in the range of $1000 \pm 500$, depending on temperature). Because of the differences in the volumetric expansion of steam and argon at different temperatures, the actual hydrodynamic parameters of the flow around the experimental basket differed at every temperature. This objectivity introduces additional variability in the apparent kinetic parameters to be obtained.

\section{Results and Discussion}

The graphs of the relative mass loss and the rise of the temperature in the middle of the basket in an argon atmosphere at three different honeycomb temperatures are presented in Figure 2. These graphs show the typical experimental data processed further by the optimization procedure to obtain the apparent kinetic parameters of pyrolysis in the described experimental setup.

The extents of pellet conversion approximated by the optimization procedure for the other experiments are presented in Figures 3, 4, 5, 6, 7, and 8.
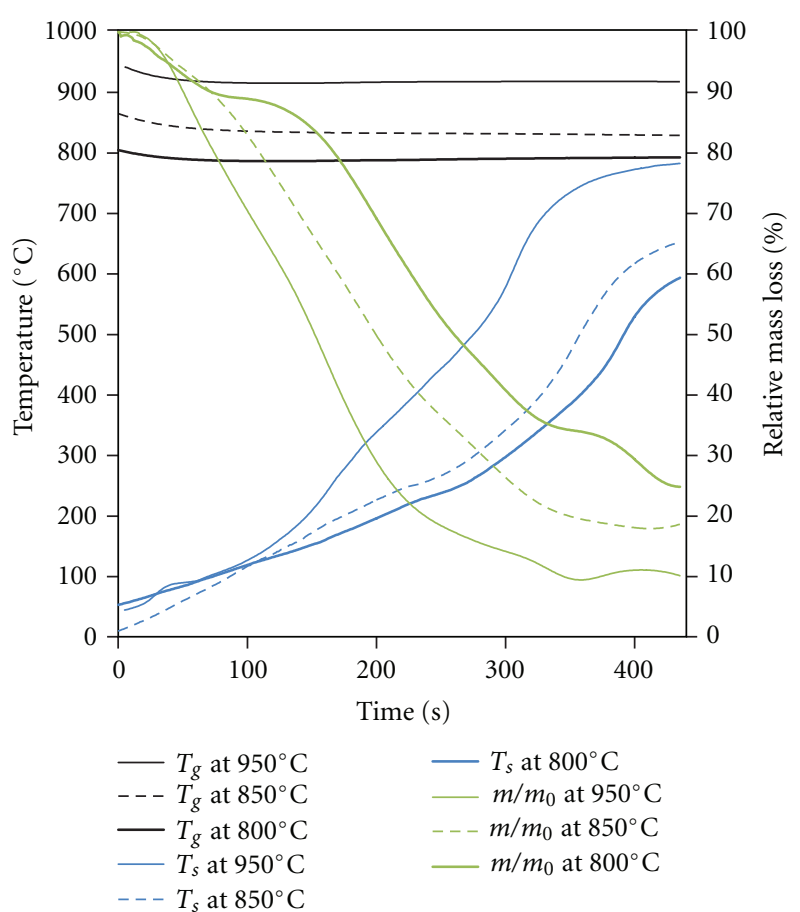

Figure 2: Temperature profiles and relative mass losses versus time for the pyrolysis of $50 \mathrm{~g}$ pellets in $1 \mathrm{Nm}^{3} / \mathrm{h}$ argon at three different temperatures $\left(T_{g}\right.$ : temperature of gas, $T_{s}$ : temperature of samples).

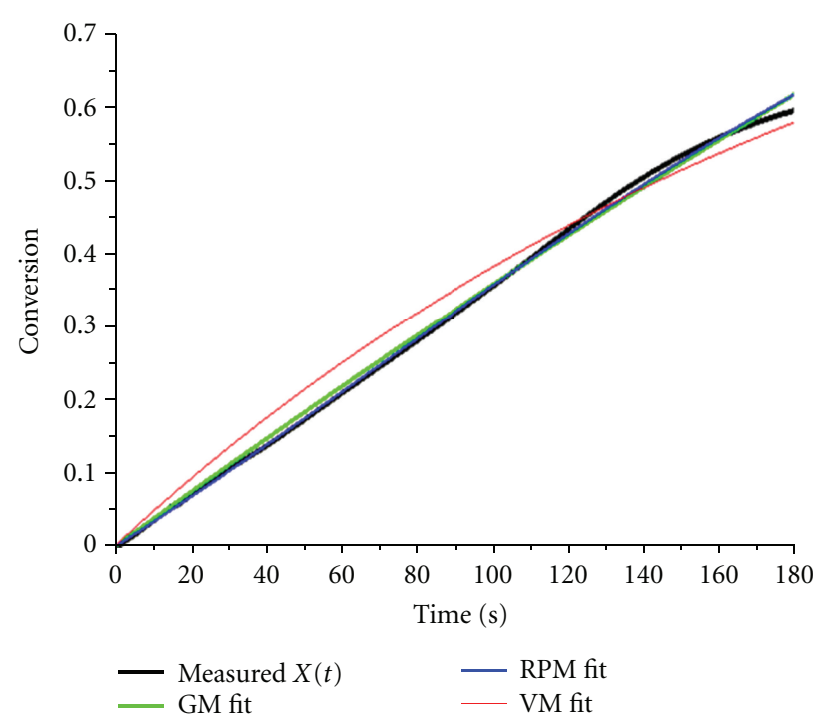

FIGURE 3: Approximated conversion of the half basket at the first temperature. $T=1065 \mathrm{~K}$; GM: form factor $=0.80, K_{0}=749 ; E_{a}=$ 100506; $R^{2}=0.998$. RPM: PSI $=4.37 ; K_{0}=164 ; E_{a}=96018 ; R^{2}=$ 0.999. VM: $K_{0}=3262 ; E_{a}=119000 ; R^{2}=0.973$.

All results of the conversion extent approximations measured in the three different series of experiments are presented in Table 2.

The table and graphs show the expected incompliance of the apparent kinetics to the Arrhenius law. This incompliance is caused by certain flaws in the hypothesis relevant to 
TABLE 2: Parameters of the pyrolysis approximations obtained with the three kinetic models.

\begin{tabular}{|c|c|c|c|c|c|c|c|c|c|c|c|c|}
\hline \multirow{2}{*}{$\mathrm{S} / \mathrm{F}$ agent } & \multirow{2}{*}{$T\left({ }^{\circ} \mathrm{C}\right)$} & \multicolumn{4}{|c|}{ RPM } & \multicolumn{4}{|c|}{ GM } & \multicolumn{3}{|c|}{$\mathrm{VM}$} \\
\hline & & $R^{2}$ & $\psi$ & $k_{0}\left(\mathrm{~s}^{-1}\right)$ & $E_{a}(\mathrm{~J} / \mathrm{Mol})$ & $R^{2}$ & $\mathrm{FF}$ & $k_{0}\left(\mathrm{~s}^{-1}\right)$ & $\begin{array}{c}E_{a} \\
(\mathrm{~J} / \mathrm{Mol})\end{array}$ & $R^{2}$ & $k_{0}\left(\mathrm{~s}^{-1}\right)$ & $E_{a}(\mathrm{~J} / \mathrm{Mol})$ \\
\hline \multirow{3}{*}{1.875} & 767 & 0.996 & 0.56 & 84 & 90023 & 0.995 & 0.33 & 82 & 90069 & 0.994 & 9367 & 130000 \\
\hline & 830 & 0.992 & 2.34 & 55 & 90024 & 0.991 & 0.33 & 63 & 90067 & 0.986 & 5381 & 130000 \\
\hline & 913 & 0.985 & 0.1 & 75 & 90020 & 0.967 & 0.33 & 58 & 90062 & 0.984 & 4508 & 130000 \\
\hline \multirow{3}{*}{3.2} & 795 & 0.985 & 0.1 & 108 & 90021 & 0.969 & 0.33 & 686 & 108012 & 0.986 & 1485 & 113000 \\
\hline & 876 & 0.993 & 10.42 & 30 & 90024 & 0.976 & 0.33 & 385 & 108037 & 0.935 & 790 & 113000 \\
\hline & 906 & 0.994 & 7.41 & 40 & 90023 & 0.975 & 0.33 & 430 & 108032 & 0.935 & 868 & 113000 \\
\hline \multirow{3}{*}{ Argon } & 788 & 0.979 & 45.08 & 504 & 114000 & 0.963 & 0.33 & 507 & 104528 & 0.938 & 950 & 109000 \\
\hline & 833 & 0.944 & 3.29 & 501 & 114000 & 0.964 & 0.85 & 452 & 104532 & 0.881 & 451 & 109002 \\
\hline & 917 & 0.996 & 11.19 & 310 & 114499 & 0.996 & 0.84 & 425 & 104532 & 0.916 & 418 & 109004 \\
\hline
\end{tabular}

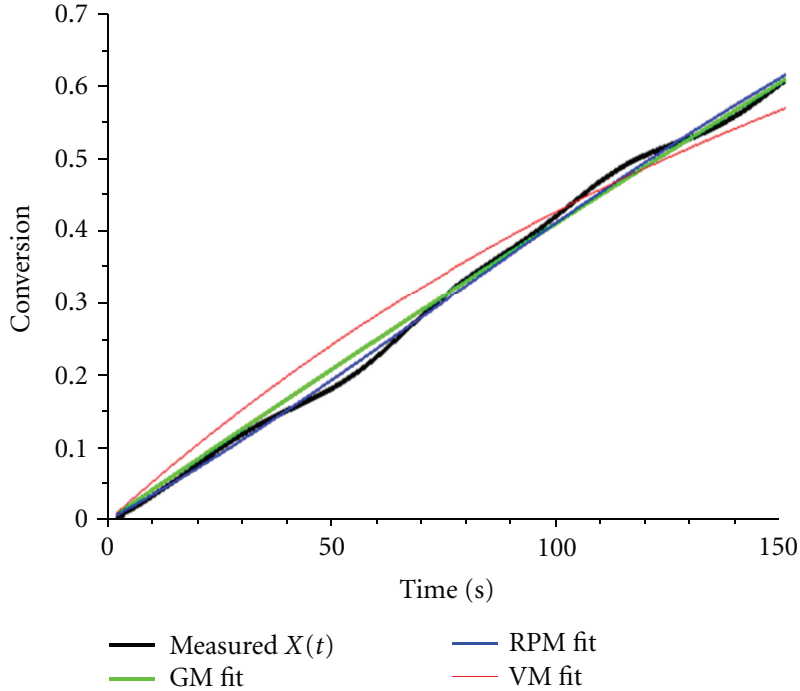

FIGURE 4: Approximated conversion of the half basket at the second temperature. $T=1158 \mathrm{~K}$; GM: form factor $=0.88 ; K_{0}=379 ; E_{a}=$ 100537; $R^{2}=0.994$. RPM: PSI $=7.13 ; K_{0}=70 ; E_{a}=96021 ; R^{2}=$ 0.997. VM: $K_{0}=1276 ; E_{a}=119000 ; R^{2}=0.961$.

the applicability of the models to larger-scale porous structures, which are changed by completely different physical processes. Pyrolysis produces not only gases but also liquids, tars, and particles, which may hinder the easy contact of the reaction-driving agents with the porous structure. However, this interference appears to be relatively small under the conditions of the high-temperature flash pyrolysis. The appearance of liquids between the pellets is not an issue at these conditions. Tars partially react with the steam in the randomly packed pellets, as we showed in [19]. The main delimiter for the easy penetration of heat to the randomly packed pellets is the active release of gaseous volatiles and tars from the pellets. This release is driven by heat, most of which is delivered by the convection of gas moving into the porous structure. The other heat transfer mechanisms, conduction and radiation, work in parallel with convection. Additional

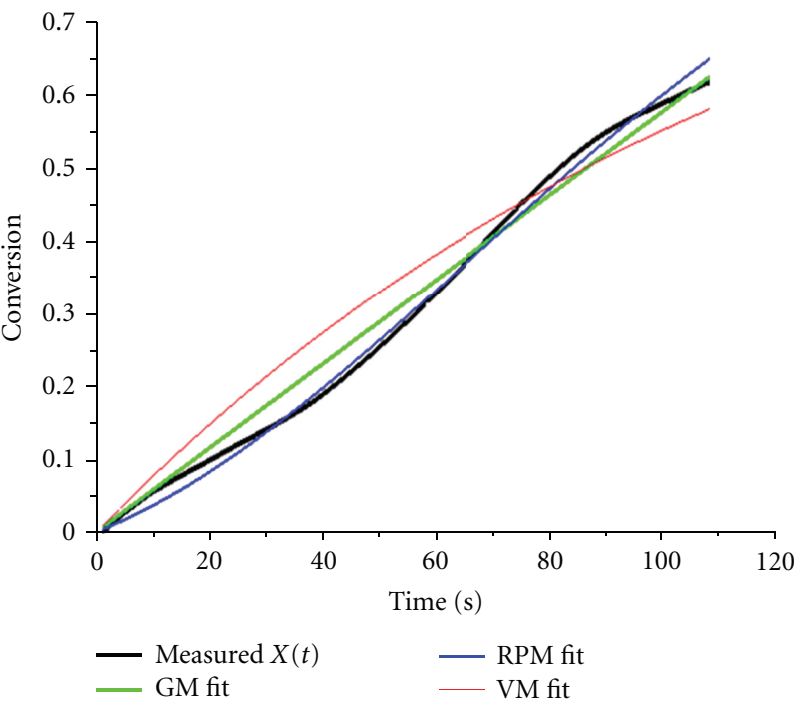

Figure 5: Approximated conversion of the half basket at the third temperature. $T=1180 \mathrm{~K}$; GM: sorm sactor $=1.00 ; K_{0}=485 ; E_{a}=$ 100527; $R^{2}=0.982$. RPM: PSI $=25.40 ; K_{0}=54 ; E_{a}=96021 ; R^{2}=$ 0.996. VM: $K_{0}=1470 ; E_{a}=119000 ; R^{2}=0.909$.

heat is transferred to the entire basket from its side because of its direct exposure to the radiation from the honey combs. The parameters describing the interplay between the different heat transfer mechanisms, practically unknown in the applied experimental conditions, do not allow exact simulation of the process. There are, however, two basic characteristics of the interplay between the transfer of an external agent driving pyrolysis (heat) and the pyrolysis itself. (1) The first characteristic is the self-regulating or self-organizing character of pyrolysis. The systems undergoing pyrolysis may be considered to be systems with self-organization because of the interplay between the process and its driving agent. As we showed in [20], the convective and radiative transfer of heat to a particle is partially delimited by the microexpansion of volatiles in the convective boundary layer around the particle. This limitation was shown by direct measurements 


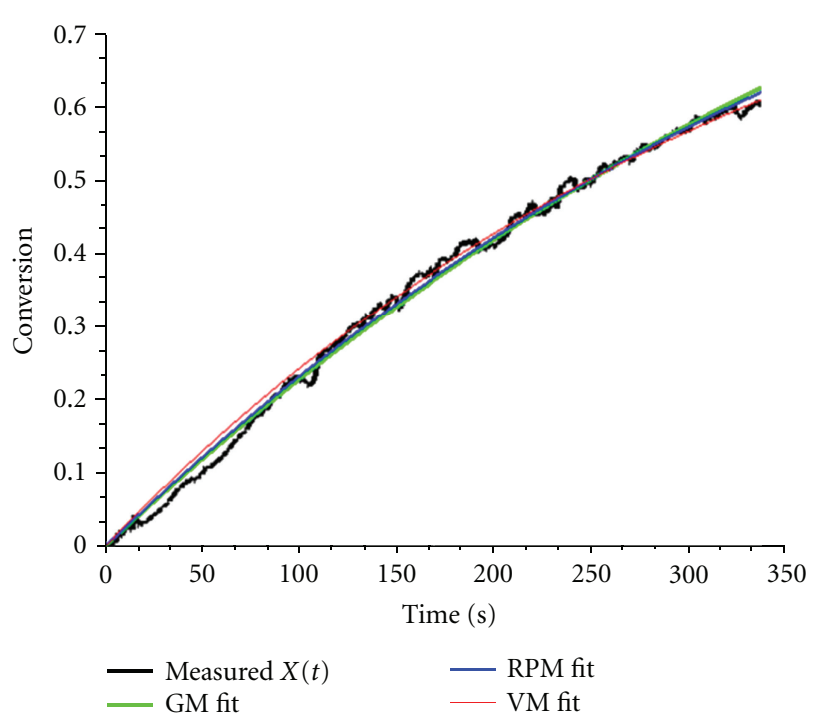

Figure 6: Approximated conversion of the full basket at the first temperature. $T=1040 \mathrm{~K}$; GM: form factor $=0.33 ; K_{0}=82 ; E_{a}=$ 90069; $R^{2}=0.995$. RPM: PSI $=0.56 ; K_{0}=84 ; E_{a}=90023 ; R^{2}=$ 0.996. VM: $K_{0}=9367 ; E_{a}=130000 ; R^{2}=0.994$.

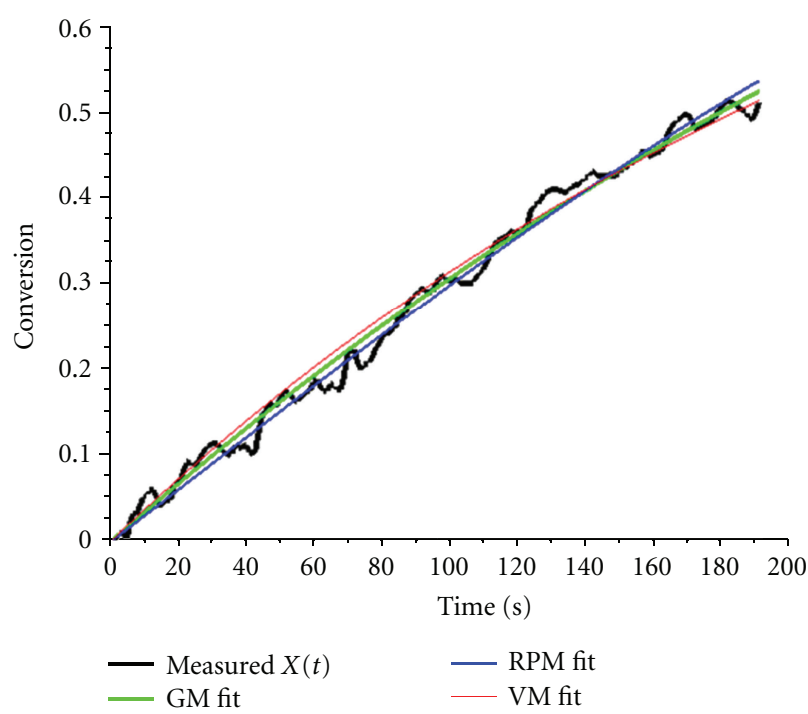

Figure 7: Approximated conversion of the full basket at the second temperature. $T=1103 \mathrm{~K}$; GM: form factor $=0.33 ; K_{0}=63 ; E_{a}=$ 90067; $R^{2}=0.991$. RPM: PSI $=2.34 ; K_{0}=55 ; E_{a}=90024 ; R^{2}=$ 0.992. VM: $K_{0}=5381 ; E_{a}=130000 ; R^{2}=0.986$.

of the temperature at the center of a relatively large particle (a wood pellet) and in proximity to its surface. The rise of the temperature near the surface of a particle is delimited by the release of volatiles during the process. In such a case, the process of externally driven heat transfer cannot be considered to be an independent controlling agent. Thus, a system under pyrolysis partially delimits the heat transfer to itself. Consequently, in the case of abundant heat sources around the particle, the control of its pyrolysis should be considered to belong to its apparent reaction kinetics. (2) The second

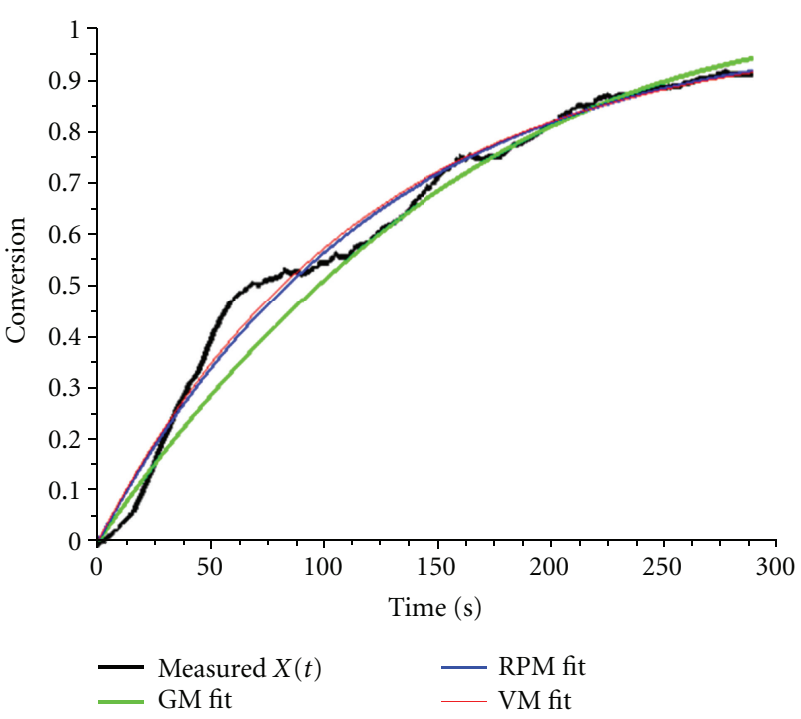

Figure 8: Approximated conversion of the full basket at the third temperature. $T=1186 \mathrm{~K}$; GM: form factor $=0.33 ; K_{0}=58 ; E_{a}=$ 90062; $R^{2}=0.967$. RPM: PSI $=0.10 ; K_{0}=75 ; E_{a}=90020 ; R^{2}=$ 0.985. VM: $K_{0}=4508 ; E_{a}=130000 ; R^{2}=0.984$.

characteristic is the dependence of the dynamics of the selforganized processes on the dynamics of the change of the porous structures. The tradeoff between the control of the internal reaction kinetics and the control of the external heat transfer may favor the latter because of the decrease of the surface of particles as their shrinkage progresses. Pyle and Zaror in [18] showed that the effect of shrinkage on the heat transfer as a result of the decreased surface of the particles is compensated by the decrease of their thickness. The progression of shrinkage may provide an increase or decrease of the overall permeability of the porous structure for both gaseous heat carriers and volatiles. Frigerio et al. [21] showed the importance of different obstacles in the flow of volatiles for the overall dynamics of pyrolysis in a fixed bed. We hypothesize that the effect of shrinkage of the entire porous structure on pyrolysis is analogous to the effects considered in the derivation of the models (RPM and GM) in their kinetic form $[2,4]$.

A somewhat unexpected flaw in the analogy may be considered because of the experimental limitations of the complexity of the entire porous structure composed of the pellets in a relatively small experimental basket. The complexity of this structure is much lower than the complexity of the microporous structure of a single biomass particle with a size less than $150 \mu \mathrm{m}$. The sizes of the micropores are in the range of angstroms, and microporosity prevails in the char [22]. Consequently, we may also apply a volumetric model to obtain the apparent kinetic parameters of the pyrolysis of such a "small" porous particle, which consists of approximately 40 or 25 pellets in the experimental conditions of this work.

It is necessary to note that the program used to obtain the apparent kinetic parameters of the models allows the selection of the parts of mass loss curves for analysis. The 
selections were made with consideration of the fact that noticeable shrinking of the porous structure in the basket does not take place during the whole process, but it occurs up to the stage of the final formation of char, as described by Lee et al. [23]. With such a selection, the approximations provided by RPM demonstrate relatively stable values of the activation energy in the range of $90 \mathrm{~kJ} / \mathrm{Mol}$. Nearly the same value was obtained with the grain model in experiments with steam. This result supports the hypothesis about the applicability of the scaling approach with both models (RPM and GM) to porous structures on scales greater than the microscale. The variability of the structural parameter, form and frequency factors of the models may be considered to be acceptable for rough predictions of the apparent pyrolysis kinetics in gasification reactors with the same internal conditions as those in the experimental basket.

The optimization procedure, which is based on measurements with argon, gave higher deviations of the parameters than the same procedure based on measurements with steam. This difference may be attributed to differences between the gases in their properties of thermal expansion, heat, and mass transfer to the basket.

If the procedure is used to select the larger parts of the conversion curves, values of the activation energies in the range of $110 \pm 20 \mathrm{~kJ} / \mathrm{mol}$ are obtained. The greatest variations in this range have been shown by a volumetric model. Because this model does not account for the presence of changing porous structures in the "gasified" particle, it is more sensitive to the peculiarities of heat and mass transfer to and from the basket.

The approximations using the extended selections of the smoothed conversion curves, which were measured for a basket with a smaller amount of pellets $(\sim 25)$ in a steam atmosphere, are presented in Figures 3, 4, and 5. The approximations of the unsmoothed conversion curves for the full basket with pellets in steam are presented in Figures 6, 7, and 8.

A remarkable characteristic of these experimental curves is their potential to be interpreted as proof of self-organization, which usually reveals itself in the self-organized oscillations [17].

The self-regulated kinetics of pyrolysis has been discussed above in the discussion about the possibility of applying the kinetic models to a process. The self-organized slow oscillations on the graphs of the measured conversion can be observed directly, and they may be more visible at the lowest temperatures using smoothing of the noisy mass-loss readings. The clearest oscillations are visible in the graph of the conversion of the full basket in the flow of steam at the highest experimental temperature in Figure 8.

This phenomenon is clearly not considered by approximations that describe the dynamic change of the porous structures in the basket during pyrolysis. The self-organization may appear because of the mechanisms mentioned above. These mechanisms consider two opposite potentials with corresponding processes, whose interaction may introduce oscillations. The first mechanism considers the potential of the pressure difference, which may simultaneously drive the mass and heat transfer into the porous structure in the basket. The opposite process appears to be caused by the pyrolysis and release of volatiles initiated by the heat transfer. The reduction of the heat transfer caused by the release of volatiles causes a decrease of the production of volatiles, followed by an increase in the heat transfer, leading to a subsequent increase in the production of volatiles and a decrease in heat transfer, in a continuous feedback loop.

The conditions necessary for such an oscillation-based regime of pyrolysis may exist because of the relatively low pressure gradients around the basket. Flows with low Reynolds numbers $(1000 \pm 500)$ may not produce large enough pressure gradients to drive the hot gas through the porous structure in a basket without noticeable resistance from the volatiles.

Another possible mechanism for the observed oscillations has been mentioned in the literature review. It provides a basic mechanism for the self-organization in any transfer process [14]. Despite the fact that the potential driving the process that opposes the transfer process is already present in the system during pyrolysis, the theory presented in [13] may provide the concept of a mechanism for the cumulative amplification of mass loss oscillations on the level of the relative microscale. Such oscillations may occur because of the interplay between the opposite potentials driving the heat transfer from a convective boundary layer into the individual pellet and the microexpansion of the pellet's volatiles moving out from its micropores into the layer. Because it is synchronized on the scale of the porous structure in the whole basket, the self-organization on the microscale level of the boundary layer of every pellet may produce the overall oscillations of the mass loss rate in the basket. The hypothetical interconnection between the interacting opposite processes on different scales may occur via the fractal geodesics introduced by Nottale in his theory.

\section{Conclusions}

The experiments and simulations of mass conversion during the pyrolysis of a changing porous structure of pellets showed the better applicability of the kinetic form of the random pore and grain models compared to the volumetric model for the partial simulation of the process.

The shrinking of the pellets during pyrolysis provides a reformation of the entire porous structure of the pellets in the random packing. This reformation changes the parameters of heat and mass transfer in and out of the structure, thus changing the dynamics of the entire process analogously to the change of the gasification dynamics in the porous structures of pellets with metallic oxides and char.

The scaling approach, which allows the application of the final expressions of the grain and random pore models in their kinetic form, changes the spatial scale to a larger one and the time scale to a shorter one (if the pyrolysis of randomly packed pellets in a basket is compared to the steam gasification of a char particle). The approach appeared to be partially applicable (up to $50-60 \%$ of the conversion curve) for a rough prediction of the apparent pyrolysis kinetics in randomly packed pellets at elevated temperatures $(T>$ $\left.700^{\circ} \mathrm{C}\right)$. 


\section{Acknowledgment}

The authors would like to express their gratitude for financial support for the experiments from EU/EIT/KIC InnoEnergy.

\section{References}

[1] B. N. Baliga, S. Dasappa, U. Shrinivasa, and H. S. Mukunda, "Gasifier-based power generation: technology and economics," Sadhana, vol. 18, no. 1, pp. 57-75, 1993.

[2] S. K. Bhatia and D. D. Perlummer, "A random pore model for fluid-solid reactions: I. Isothermal, kinetic control," AIChE Journal, vol. 26, no. 3, pp. 379-385, 1980.

[3] S. K. Bhatia and D. D. Perlmutter, "A random pore model for fluid-solid reactions: II. Diffusion and transport effects," AIChE Journal, vol. 27, no. 2, pp. 247-254, 1981.

[4] J. Szekely and J. W. Evans, "A structural model for gas-solid reactions with a moving boundary," Chemical Engineering Science, vol. 25, no. 6, pp. 1091-1107, 1970.

[5] J. Szekely and J. W. Evans, "A structural model for gas-solid reactions with a moving boundary-II. The effect of grain size, porosity and temperature on the reaction of porous pellets," Chemical Engineering Science, vol. 26, no. 11, pp. 1901-1913, 1971.

[6] J. Fermoso, B. Arias, C. Pevida, M. G. Plaza, F. Rubiera, and J. J. Pis, "Kinetic models comparison for steam gasification of different nature fuel chars," Journal of Thermal Analysis and Calorimetry, vol. 91, no. 3, pp. 779-786, 2008.

[7] K. Raghunathan and R. Y. K. Yang, "Unification of coal gasification data and its applications," Industrial and Engineering Chemistry Research, vol. 28, no. 5, pp. 518-523, 1989.

[8] T. Yamashita, Y. Fujii, Y. Morozumi, H. Aoki, and T. Miura, "Modeling of gasification and fragmentation behavior of char particles having complicated structures," Combustion and Flame, vol. 146, no. 1-2, pp. 85-94, 2006.

[9] H. Wu, K. Yip, F. Tian, Z. Xie, and C. Z. Li, "Evolution of char structure during the steam gasification of biochars produced from the pyrolysis of various mallee biomass components," Industrial and Engineering Chemistry Research, vol. 48, no. 23, pp. 10431-10438, 2009.

[10] B. H. Song and S. D. Kim, "Catalytic activity of alkali and iron salt mixtures for steam-char gasification," Fuel, vol. 72, no. 6, pp. 797-803, 1993.

[11] X. Wang, H. Zhang, and L. Zheng, "Novel modeling tool for fixed-bed biomass gasification using high-temperature air," Industrial and Engineering Chemistry Research, vol. 46, no. 26, pp. 8852-8856, 2007.

[12] R. Gutfraind and M. Sheintuch, "Scaling approach to study diffusion and reaction processes on fractal catalysts," Chemical Engineering Science, vol. 47, no. 17-18, pp. 4425-4433, 1992.

[13] L. Nottale, "Scale relativity and fractal space-time: theory and applications," in Proceedings of the 1st International Conference on the Evolution and Development of the Universe, pp. 101-152, Foundation of Science, Paris, France, 2008.

[14] L. Notalle, "Generalized quantum potentials," Journal of Physics A, vol. 92, no. 10, pp. 2068-2074, 2011.

[15] A. Alevanau, I. Ahmed, A. K. Gupta, W. Yang, and W. Blasiak, "Parameters of high temperature steam gasification of original and pulverised wood pellets," Fuel Processing Technology, vol. 92, no. 10, pp. 2068-2074, 2011.
[16] J. Feder, Fractals, Plenum Press, New York, NY, USA, 1991.

[17] H. Haken, Synergetics, an Introduction: nonequilibrium Phase Transitions and Self-Organization in Physics, Chemistry, and Biology, Springer, 3rd edition, 1983.

[18] D. L. Pyle and C. A. Zaror, "Heat transfer and kinetics in the low temperature pyrolysis of solids," Chemical Engineering Science, vol. 39, no. 1, pp. 147-158, 1984.

[19] P. Donaj, W. Yang, and W. Blasiak, "Conversion of industrialy processed biomas waste into value-added products using high temperature agents," in Proceedings of the International Conference on Thermal Treatment Technologies and Hazardous Waste Combustors (IT3/HWC '11), Jacksonville, Fla, USA, 2011.

[20] A. Alevanau, E. Kantarelis, W. Yang, and W. Blasiak, "Study of the effects of gaseous micro-expansion on the efficiency of convective heat transfer during pyrolysis," Submitted to Fuel Processing Technology.

[21] S. Frigerio, H. Thunman, B. Leckner, and S. Hermansson, "Estimation of gas phase mixing in packed beds," Combustion and Flame, vol. 153, no. 1-2, pp. 137-148, 2008.

[22] S. Link, S. Arvelakis, M. Hupa, P. Yrjas, I. Külaots, and A. Paist, "Reactivity of the biomass chars originating from reed, douglas fir, and pine," Energy and Fuels, vol. 24, no. 12, pp. 6533-6539, 2010.

[23] C. K. Lee, R. F. Chaiken, and J. M. Singer, "Charring pyrolysis of wood in fires by laser simulation," Symposium (International) on Combustion, vol. 16, no. 1, pp. 1459-1470, 1977. 

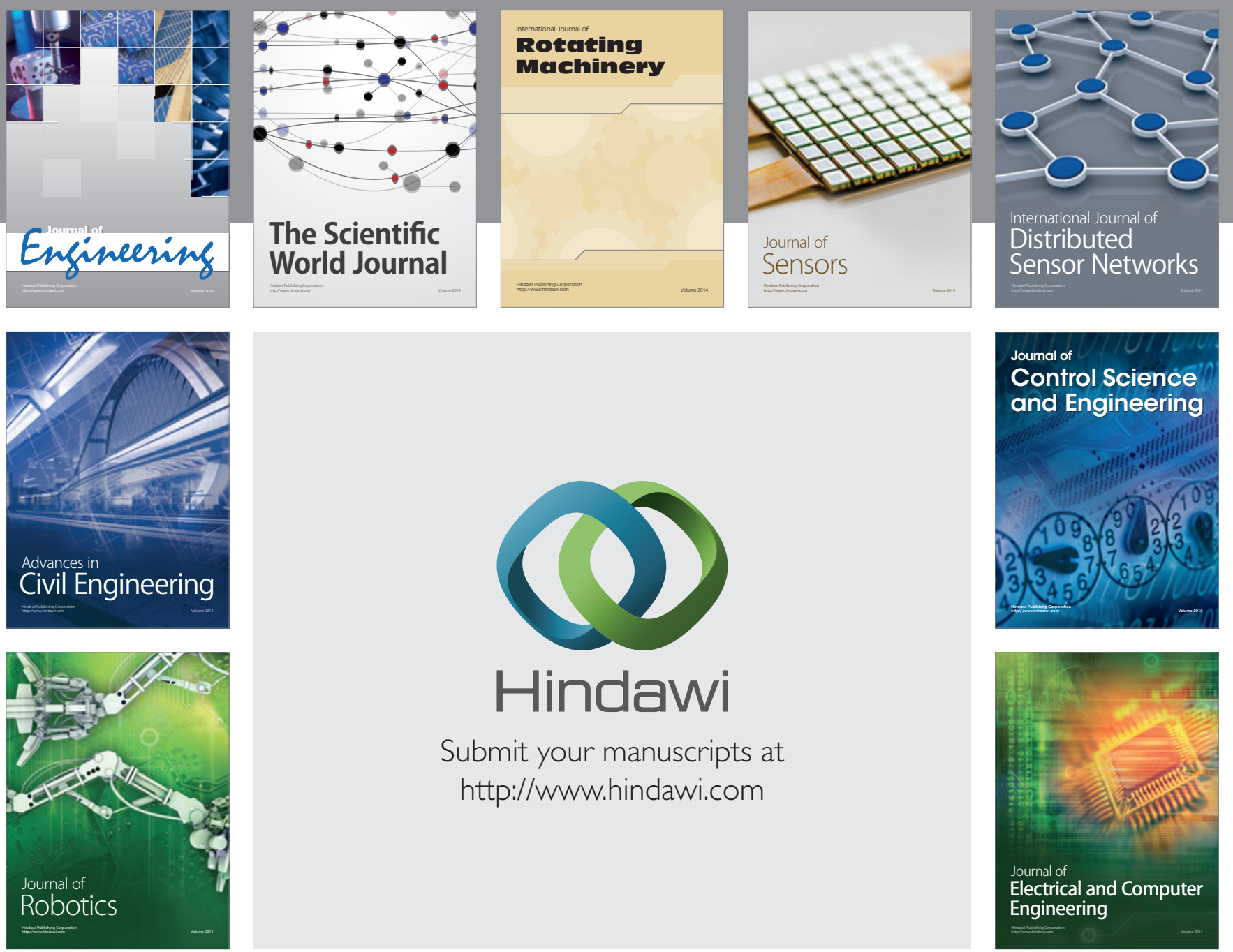

Submit your manuscripts at

http://www.hindawi.com
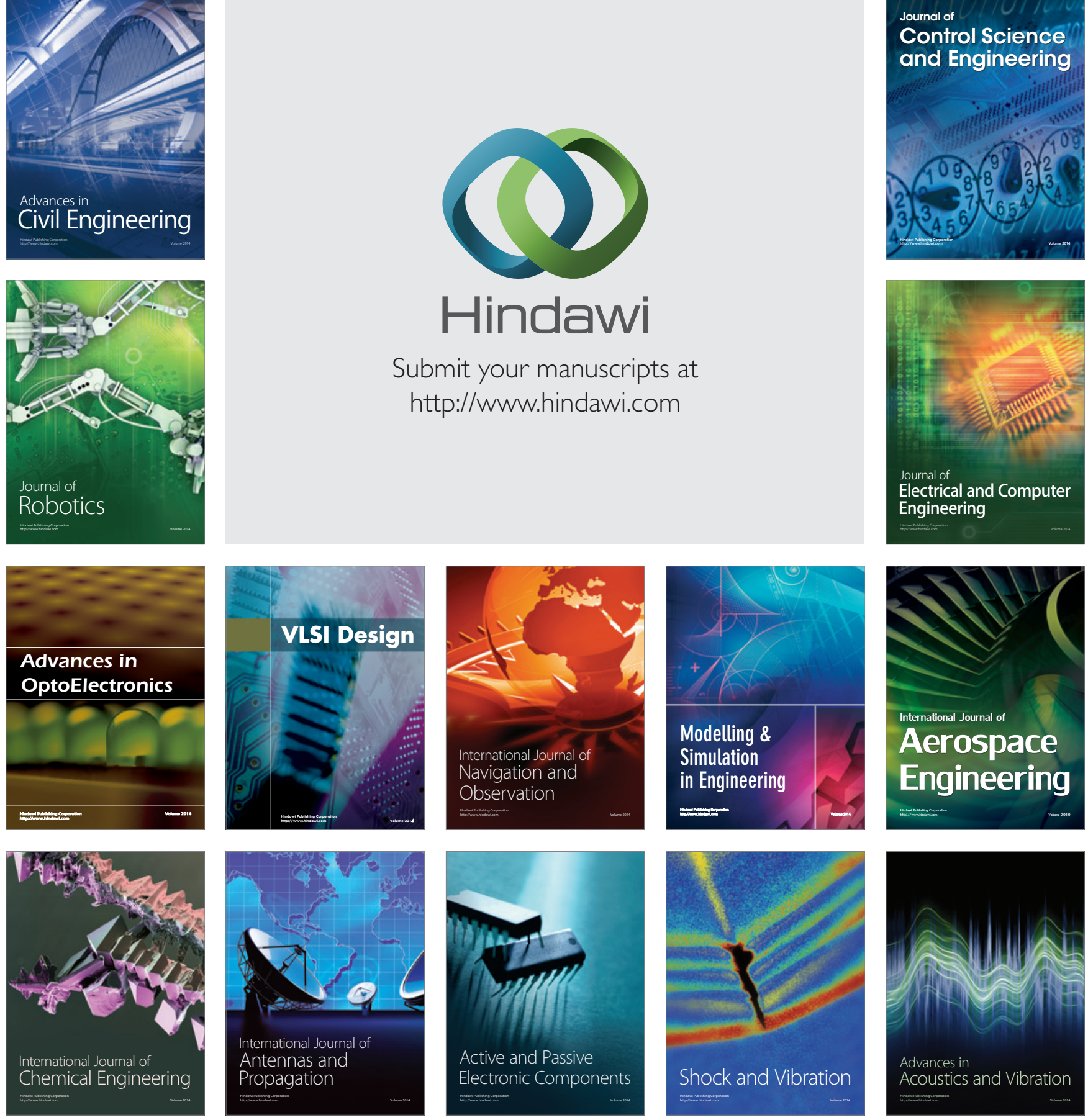